\title{
Effect of Obesity-Linked FTO rs9939609 Variant on Physical Activity and Dietary Patterns in Physically Active Men and Women
}

\author{
Nathan R. West $\mathbb{D}^{1},{ }^{1}$ James Dorling, ${ }^{2}$ Alice E. Thackray $\mathbb{D}^{2}{ }^{2}$ Nicola C. Hanson $\left(\mathbb{D},{ }^{1}\right.$ \\ Samantha E. Decombel $\left(\mathbb{D},{ }^{1}\right.$ David J. Stensel $\left(\mathbb{D},{ }^{2}\right.$ and Stuart J. Grice $\mathbb{D}^{1,3}$ \\ ${ }^{1}$ FitnessGenes, Bicester Innovation Centre, Commerce House, Telford Rd., Bicester OX26 4LD, UK \\ ${ }^{2}$ School of Sport, Exercise and Health Sciences, Loughborough University, Loughborough, Leicestershire LE11 3TU, UK \\ ${ }^{3}$ MRC Functional Genomics Unit, Department of Physiology, Anatomy and Genetics, University of Oxford, South Parks Road, \\ Oxford OX1 3PT, UK
}

Correspondence should be addressed to Stuart J. Grice; stuartjfgrice@gmail.com

Received 28 June 2017; Revised 10 November 2017; Accepted 26 November 2017; Published 1 March 2018

Academic Editor: R. Prager

Copyright (c) 2018 Nathan R. West et al. This is an open access article distributed under the Creative Commons Attribution License, which permits unrestricted use, distribution, and reproduction in any medium, provided the original work is properly cited.

\begin{abstract}
Single nucleotide polymorphisms (SNPs) in the fat mass and obesity-associated (FTO) locus are associated with obesity, but lifestyle factors may modulate the obesity risk related to FTO. This study examined the physical activity and dietary patterns of 528 physically active white men and women (mean (SD): 34.9 (9.5) years, $26.6(4.3) \mathrm{kg} \cdot \mathrm{m}^{-2}$ ) carrying different risk variants of FTO SNP rs9939609. Sex, age, and anthropometric measurements (stature, body mass, and waist circumference) were self-reported using an online questionnaire, and body mass index and waist-to-height ratio were calculated. Physical activity and eating behaviour were assessed using the International Physical Activity Questionnaire (IPAQ) and Three-Factor Eating Questionnaire (TFEQ), respectively. Body mass, body mass index (BMI), waist circumference, and waist-to-height ratio were not significantly different between individuals expressing different FTO rs9939609 risk variants (all $P \geq 0.66$ ). The cohort was physically active (4516 (3043) total METmin.week ${ }^{-1}$ ), although homozygous risk allele carriers (AA) displayed higher TFEQ cognitive restraint compared with nonrisk allele carriers $(\mathrm{TT})(\mathrm{ES}=0.33$ and $P=0.03)$. In conclusion, obesity-related parameters were not different in physically active individuals expressing different risk variants of FTO rs9939609, although homozygous risk allele carriers exhibited higher cognitive restraint.
\end{abstract}

\section{Introduction}

Obesity is a major risk factor for several chronic diseases and represents a major health and economic burden on society [1]. The aetiology of obesity is multifactorial and is influenced by complex interactions between environmental, lifestyle, and genetic factors [2]. Consequently, it is important to understand the interplay between these factors when designing strategies targeting the prevention of obesity.

The fat mass and obesity-associated gene (FTO) was the first common variant identified by genome-wide association studies that influences obesity risk [3]. Single nucleotide polymorphisms (SNPs) in intron 1 of FTO have been associated consistently with obesity risk across different ages and populations [4-9]. At FTO rs9939609 SNP, individuals homozygous for the risk allele (AA) weigh $3 \mathrm{~kg}$ more and have a 1.7-fold higher risk of obesity than those who do not carry a risk allele (TT) [3]. Studies have examined the effect of FTO variants on regulators of energy homeostasis to elucidate the mechanisms influencing FTO-mediated obesity risk. In this respect, evidence suggests that FTO may play a central role in the regulation of food intake [10, 11]. This is supported by studies demonstrating that individuals homozygous for the risk allele exhibit reduced satiety, poor food choices, and increased energy consumption [12-14]. Conversely, FTO obesity SNPs have not been related to energy expenditure, with evidence suggesting that those 
TABLE 1: Participant characteristics.

\begin{tabular}{|c|c|c|c|}
\hline & Total $(n=528)$ & Female $(n=107)$ & Male $(n=421)$ \\
\hline \multicolumn{4}{|c|}{ Age and anthropometric characteristics } \\
\hline Age (years) & $34.9(9.5)$ & $36.8(9.1)$ & $34.5(9.6)^{*}$ \\
\hline Stature $(\mathrm{cm})$ & $176.5(9.4)$ & $164.9(7.1)$ & $179.5(7.4)^{*}$ \\
\hline Body mass $(\mathrm{kg})$ & $83.4(16.5)$ & $69.0(13.9)$ & $87.0(15.0)^{*}$ \\
\hline Body mass index $\left(\mathrm{kg} \cdot \mathrm{m}^{-2}\right)$ & $26.6(4.3)$ & $25.4(4.8)$ & $27.0(4.0)^{*}$ \\
\hline Waist circumference $(\mathrm{cm})$ & $84.6(10.4)$ & $78.2(11.8)$ & $86.2(9.4)^{*}$ \\
\hline Waist-to-height ratio & $0.48(0.06)$ & $0.48(0.07)$ & $0.48(0.05)$ \\
\hline Central obesity ${ }^{\mathrm{a}}$ & $37(7 \%)$ & $21(20 \%)$ & $16(4 \%)^{* *}$ \\
\hline \multicolumn{4}{|l|}{ Physical activity levels ${ }^{\mathrm{b}}$} \\
\hline Vigorous MET (min·week ${ }^{-1}$ ) & $2629(1908)$ & $2511(1912)$ & $2659(1908)$ \\
\hline Moderate MET (min.week ${ }^{-1}$ ) & $737(1026)$ & $716(874)$ & $743(1062)$ \\
\hline Walking MET (min·week ${ }^{-1}$ ) & $1150(1269)$ & $1284(1374)$ & $1115(1241)$ \\
\hline Total MET (min'week ${ }^{-1}$ ) & $4516(3043)$ & $4511(3163)$ & $4518(3017)$ \\
\hline \multicolumn{4}{|l|}{ Eating behaviour } \\
\hline Cognitive restraint score & $12(4)$ & $12(4)$ & $12(4)$ \\
\hline Disinhibition score & $6(4)$ & $7(4)$ & $6(3)^{*}$ \\
\hline Hunger score & $5(4)$ & $6(4)$ & $5(4)$ \\
\hline \multicolumn{4}{|l|}{ FTO rs99396909 genotype } \\
\hline AA & $95(18 \%)$ & $18(17 \%)$ & $77(18 \%)$ \\
\hline $\mathrm{AT}$ & $236(45 \%)$ & $40(37 \%)$ & $196(47 \%)$ \\
\hline TT & $197(37 \%)$ & $49(46 \%)$ & $148(35 \%)$ \\
\hline
\end{tabular}

Values for age, anthropometric characteristics, physical activity levels, and eating behaviour are mean (SD) and were analysed between sex using linear mixed models. Values for central obesity and FTO rs 99396909 genotype are frequency (\%) and were analysed between sex using chi-square tests. ${ }^{\mathrm{a} C e n t r a l}$ obesity was defined as a waist circumference $>88 \mathrm{~cm}$ for women and $>102 \mathrm{~cm}$ for men. ${ }^{\text {b } C o m p l e t e ~ p h y s i c a l ~ a c t i v i t y ~ d a t a ~ a v a i l a b l e ~ f o r ~} n=408$ (female: $n=83$; male: $n=325) .{ }^{*}$ Significant difference between females and males (linear mixed model $\left.P<0.05\right) .{ }^{* *}$ Significant difference between females and males (chi-square test $P<0.05)$.

carrying the risk allele do not show reduced basal metabolic rate [15] or physical activity levels [7, 16, 17].

Although the association between FTO and obesity risk is well established, lifestyle may modulate obesity risk related to FTO. Several studies have suggested that FTO-mediated body fatness may be attenuated in physically active individuals [18-20]. Indeed, a meta-analysis concluded that higher physical activity levels attenuate the influence of FTO variation on obesity risk by $30 \%$ [21], and exercise interventions have demonstrated efficacy in promoting weight loss in FTO risk allele carriers [22, 23]. However, it is not well understood how the body mass index- (BMI-) increasing influence of FTO is attenuated in physically active individuals. An improved understanding of the differences in dietary and physical activity patterns in variants of FTO rs9939609 SNP within a physically active cohort may provide a greater insight into the behaviours that offset FTOmediated obesity. Therefore, the aim of this study was to examine physical activity and dietary habits in a sample of physically active men and women carrying different risk variants of FTO rs9939609 SNP.

\section{Materials and Methods}

2.1. Participants. With the approval of Loughborough University's Ethical Advisory Committee, 708 men and women (age $34.0( \pm 9.3)$ years; body mass $82.5( \pm 16.3) \mathrm{kg}$;
BMI $26.5( \pm 4.2) \mathrm{kg} \cdot \mathrm{m}^{-2}$; waist circumference $84.4( \pm 11.0)$ $\mathrm{cm}$; and waist-to-height ratio $0.48( \pm 0.06))$ were recruited between March to November 2015 to participate in this study. Participants were recruited through FitnessGenes Ltd. (Bicester, Oxfordshire, UK-previously MuscleGenes Ltd.) and were predominantly based in European countries or the United States. Full informed consent, via an online consent form, was obtained from participants before the study commenced. Participants were volunteers from a customer base that were known to be participating in a regular fitness program but were not involved in elite sports. Participants classified themselves into one of fifteen ethnic groups. To reduce variability in the cohort, individuals who identified themselves as non-mixed white descent (British, Irish, and other White background) were included in this study (see Supplementary Methods (available here) for ethnic background selection criteria). Of the 708 recruited, 1 had missing genotype data, 17 had missing demographic or anthropometric data, and 162 did not classify themselves as non-mixed white descent. Therefore, results are presented for 528 participants (421 males and 107 females). Participant characteristics are presented in Table 1.

2.2. Genotype Analysis. Participant DNA was obtained from saliva, which was collected via an Oragene DNA selfcollection kit (DNA Genotek Inc., Ottawa, ON, Canada) 
sent from and returned to FitnessGenes Ltd. by post. DNA was extracted by LGC Genomics (Hertfordshire, UK) and genotyped using a $\mathrm{KASP}^{\mathrm{TM}}$ genotyping assay for FTO rs9939609 SNP. Genotype frequency of FTO rs9939609 SNP was assessed using a goodness-of-fit chi-square test and did not deviate from Hardy-Weinberg equilibrium $(\mathrm{AA}=95$, $\mathrm{AT}=236, \mathrm{TT}=197 ; P=0.10)$.

\subsection{Collection of Demographic and Anthropometric} Data. Participants self-reported their sex, age, stature, body mass, waist circumference, country of birth, and ethnicity using an online questionnaire. Sex, age, and ethnicity were confirmed by cross-referencing against customer information held by FitnessGenes Ltd. Participant stature, body mass, and waist circumference were used to calculate BMI and waist-to-height ratio (Table 1). Central obesity was calculated as the percentage of participants that exceeded previously defined thresholds for waist circumference (females $>88 \mathrm{~cm}$ and males $>102 \mathrm{~cm}$ ) [24].

2.4. Physical Activity Levels. Physical activity levels were measured using the validated short format of the International Physical Activity Questionnaire (IPAQ) [25]. The IPAQ assesses the frequency and duration of walking, and moderate- and vigorous-intensity physical activities performed in bouts lasting 10 minutes or more during the previous seven days. Total physical activity (MET (metabolic equivalent), minutes per week) was estimated by multiplying the number of minutes reported in each activity level by the specific MET score for that activity (walking: 3.3, moderate intensity: 4.0, and vigorous intensity: 8.0 METs), and participants were classified in one of the three physical activity levels: low, moderate, or high (http://www.ipaq.ki.se).

2.5. Eating Behaviour. Eating behaviour was assessed using the validated 51-item Three-Factor Eating Questionnaire (TFEQ) to measure the dietary restraint (21 items, Cronbach's $\alpha$ 0.788), disinhibition (16 items, Cronbach's $\alpha 0.745$ ), and hunger (14 items, Cronbach's $\alpha$ 0.761) [26]. All TFEQ items were coded with either 0 or 1 point and summed within each domain. Higher scores within each domain were indicative of higher restrained eating, disinhibited eating, or a predisposition to hunger.

2.6. Statistical Analyses. Data were analysed using the IBM SPSS Statistics Software for Windows Version 21 (IBM, New York). Between-sex differences in participant characteristics were examined using linear mixed models with one fixed factor (sex). Linear mixed models, adjusted for age and sex, were used to examine between-genotype differences in obesity-related parameters, physical activity levels, and eating behaviour with one fixed factor (FTO rs99396909 genotype). Between-genotype differences in all outcome measures were analysed using the additive genotype model (AA versus AT versus TT). Statistical power for this model was estimated using the CaTS Genome Power Calculator methods [27]. This method calculates the probability of outcomes per genotype, and thus the statistical power of the study based on the disease allele frequencies and prevalence of the disease within a population (i.e., the probability that a randomly sampled individual is obese or has a higher BMI) [27]. The calculation was performed using the genotype relative risk (GRR) for the additive genotype model (GRR $=f_{1} / f_{0}$, where $f_{0}$ and $f_{1}$ are the probabilities of being affected for individuals with 0 or 1 risk allele, resp.). For this study, a power of over 0.97 for a one-stage study was reported with a significance of 0.005 . Where significant main effects were found in the additive genotype model, post hoc analysis was performed using Holm-Bonferroni correction for multiple comparisons [28]. Exploratory analysis was also conducted to analyse potential between-genotype differences in all outcome measures using the dominant model (risk allele carriers (AA/AT) versus homozygous nonrisk genotype (TT)) and the recessive model (nonrisk allele carriers (AT/TT) versus homozygous risk genotype (AA)). Differences in categorical variables (central obesity and FTO genotype frequency) between sex and/or FTO genotype groups were analysed using chi-square tests. Effect sizes are used to supplement important findings. An effect size of 0.2 was considered the minimum important difference in all outcome measures, 0.5 was moderate, and 0.8 was large [29]. Continuous variables are presented as mean (SD) and categorical variables as frequency (\%). Statistical significance was accepted as $P<0.05$.

\section{Results}

3.1. Participant Characteristics. On average, participants reported engaging in a total of 4516 (3043) MET minutes of activity per week, and the majority of participants were classified in the high physical activity category of the IPAQ (high $75.2 \%$, moderate $19.4 \%$, and low 5.4\%), confirming the cohort were physically active (Table 1). Moreover, participants reported engaging in, on average, 3366 MET min.week ${ }^{-1}$ of moderate- to vigorous-intensity physical activity (Table 1), which is considerably higher than the average levels of moderate- to vigorous-intensity physical activity reported in adults across European countries (range 45-960 METmin·week ${ }^{-1}$ ) [30]. Participants reported the following reasons for engaging in physical activity: muscle building training $n=276$ (52\%); fat loss training $n=168$ (32\%); strength training $n=40(8 \%)$; power training $n=20$ (4\%); endurance training $n=17(3 \%)$; and no response $n=7(1 \%)$.

Females were significantly older $(\mathrm{ES}=0.24, P=0.02)$, had a higher frequency of central obesity (odds ratio $=6.31$, $P<0.001)$, and exhibited higher TFEQ disinhibition scores (ES $=0.42, P<0.001$ ) compared with males (Table 1 ). Body mass, BMI, and waist circumference were significantly lower in females than males (all ES $\geq 0.38, P \leq 0.001$ ), but waist-toheight ratio was similar between the sexes $(E S=0.09$, $P=0.40$ ) (Table 1). No significant differences were seen between the sexes for any measure of physical activity (all $\mathrm{ES} \leq 0.13, P \geq 0.28)$ or FTO genotype frequency $(P=0.12)$ (Table 1). 


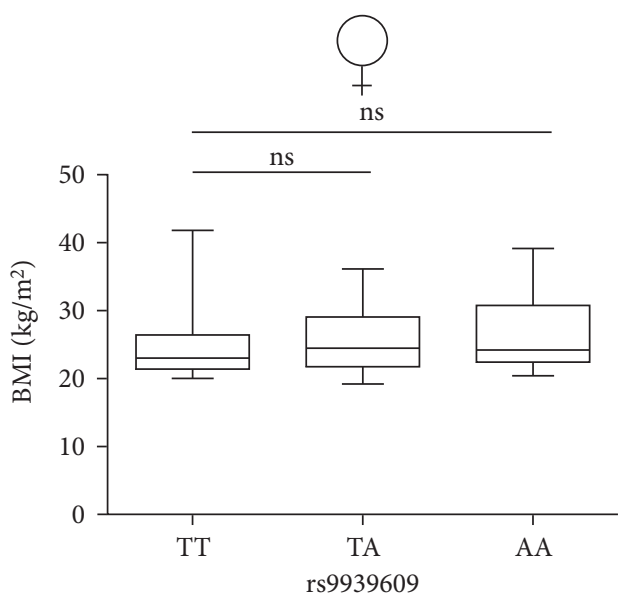

(a)

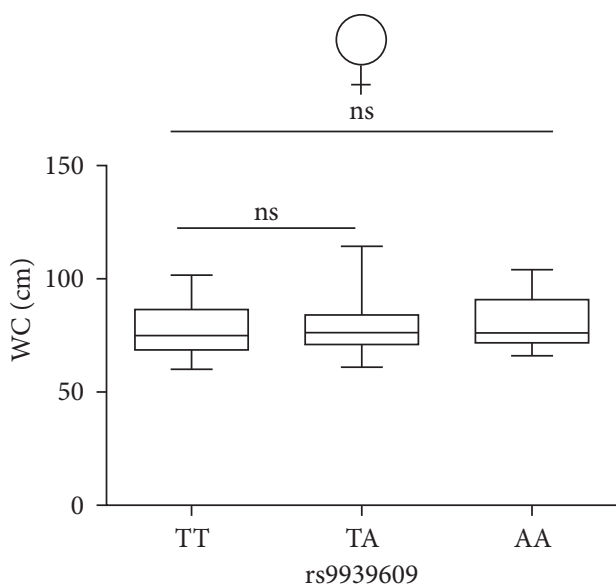

(c)

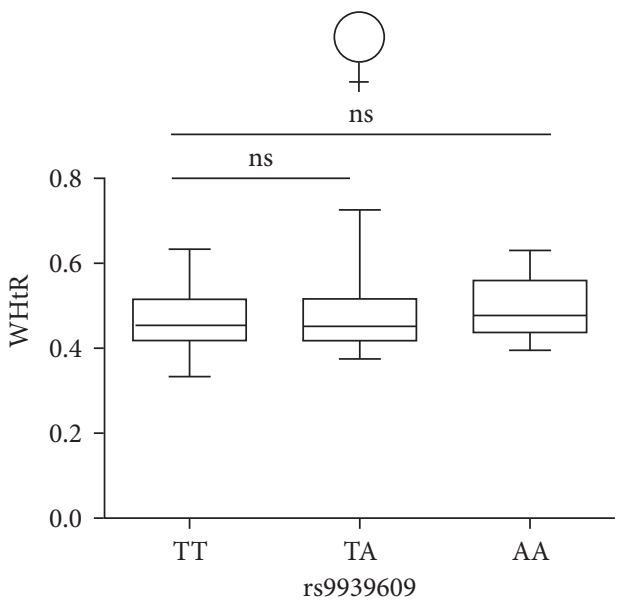

(e)

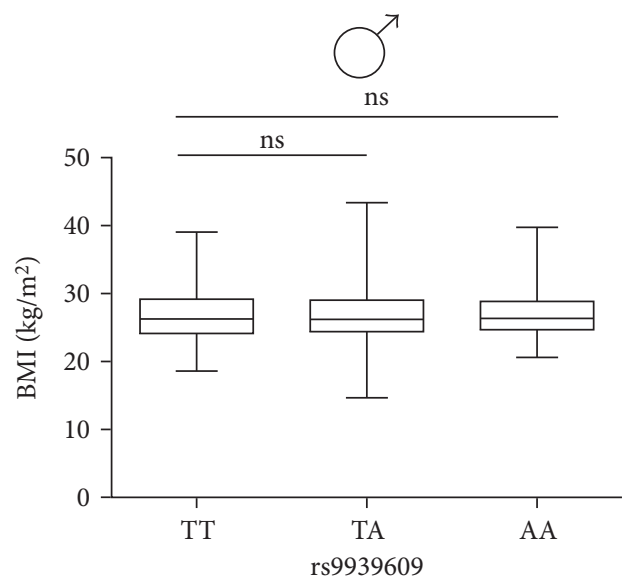

(b)

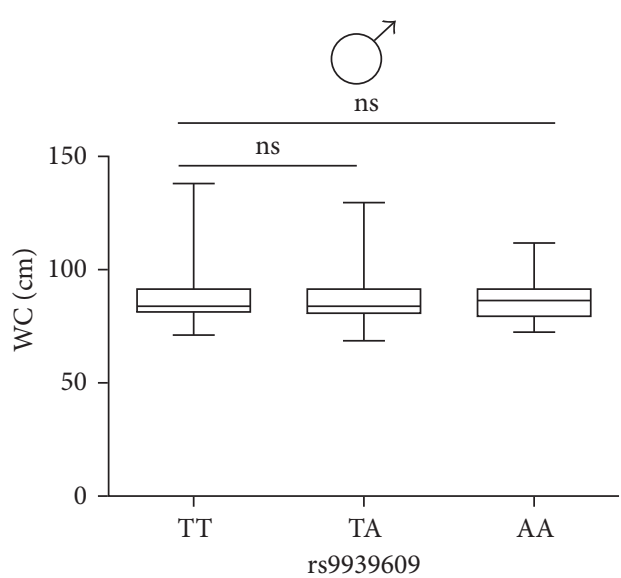

(d)

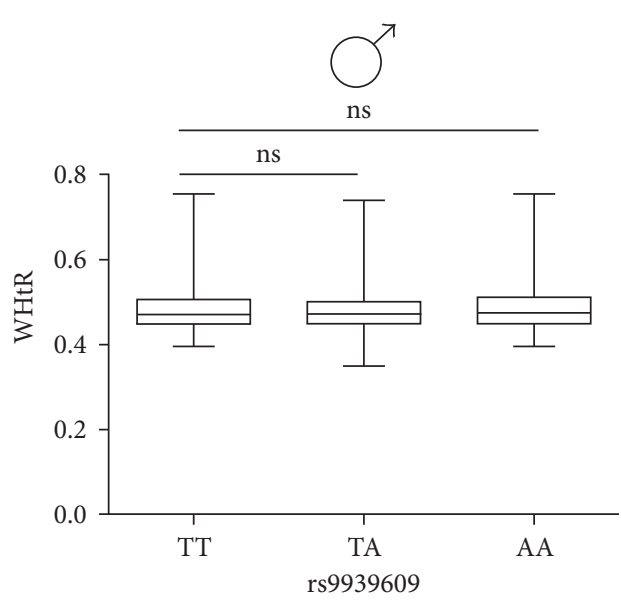

(f)

Figure 1: Box plots and analysis of the effect of FTO rs9939609 gene variant on obesity-related parameters. (a, b) Body mass index (BMI); (c, d) waist circumference (WC); (e, f) waist-to-height ratio (WHtR). (a, c, e) female; (b, d, f) male. Females: $n=107$ (AA = 18, AT = 40, and $\mathrm{TT}=49)$. Males: $n=421$ (AA = 77, AT =196, and TT =148). The line within box represents the median, while the lower and upper box lines represent the interquartile range (1st and 3rd quartiles). The whiskers represent the minimum and maximum observations. Linear mixed models, adjusted for age and sex, were used to examine between-genotype differences. No significant differences were seen in obesity-related parameters between FTO rs 9939609 genotypes. 
TABLE 2: Obesity-related parameters in men and women carrying different risk variants of the FTO rs9939609 single nucleotide polymorphism.

\begin{tabular}{|c|c|c|c|c|}
\hline \multirow{2}{*}{ Characteristic } & \multicolumn{3}{|c|}{ FTO rs9939609 genotype } & \multirow{2}{*}{ Model $1^{\text {a }}$ : genotype $P$ value } \\
\hline & AA & AT & TT & \\
\hline Body mass (kg) & $83.7(14.7)$ & $84.4(16.5)$ & $82.0(17.2)$ & 0.69 \\
\hline Body mass index $\left(\mathrm{kg} \cdot \mathrm{m}^{-2}\right)$ & $26.8(3.9)$ & $26.8(4.3)$ & $26.4(4.3)$ & 0.66 \\
\hline Waist circumference $(\mathrm{cm})$ & $85.2(9.4)$ & $84.9(10.4)$ & $84.1(11.0)$ & 0.87 \\
\hline Waist-to-height ratio & $0.48(0.05)$ & $0.48(0.06)$ & $0.48(0.06)$ & 0.85 \\
\hline Central obesity ${ }^{\mathrm{b}}$ & $6(7 \%)$ & $16(7 \%)$ & $15(8 \%)$ & 0.92 \\
\hline
\end{tabular}

Values for body mass, body mass index, waist circumference, and waist-to-height ratio represent mean (SD) and were analysed using linear mixed models adjusted for age and sex. Values for central obesity represent frequency (\%) and were analysed using chi-square tests. ES, effect size (body mass, body mass index, waist circumference, and waist-to-height ratio); OR, odds ratio (central obesity). ${ }^{\text {a }}$ Model 1: additive genotype model (AA versus AT versus TT).

${ }^{\mathrm{b}}$ Central obesity was defined as a waist circumference $>88 \mathrm{~cm}$ for women and $>102 \mathrm{~cm}$ for men.

TABLE 3: Physical activity levels in men and women carrying different risk variants of the FTO rs9939609 single nucleotide polymorphism.

\begin{tabular}{lcccc}
\hline Characteristic & \multicolumn{3}{c}{ FTO rs9939609 genotype } & Model $1^{\text {a }}$ : genotype $P$ value \\
\hline Vigorous MET $\left(\mathrm{min} \cdot \mathrm{week}^{-1}\right)$ & $2808(1966)$ & $2751(1903)$ & $2407(1876)$ & 0.19 \\
Moderate MET $\left(\mathrm{min} \cdot \mathrm{week}^{-1}\right)$ & $726(1069)$ & $816(1127)$ & $654(874)$ & 0.41 \\
Walking MET $\left(\mathrm{min} \cdot \mathrm{week}^{-1}\right)$ & $1240(1242)$ & $1207(1345)$ & $1043(1192)$ & 0.34 \\
Total MET $\left(\mathrm{min} \cdot \mathrm{week}^{-1}\right)$ & $4774(3125)$ & $4774(3193)$ & $4104(2795)$ & 0.10 \\
\hline
\end{tabular}

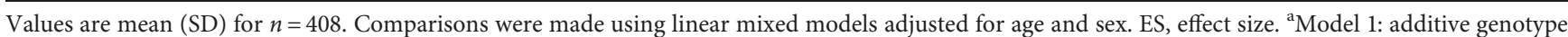
model (AA versus AT versus TT).

TABLE 4: Eating behaviour in men and women carrying different risk variants of the FTO rs9939609 single nucleotide polymorphism.

\begin{tabular}{lcccc}
\hline Characteristic & & FTO rs9939609 genotype & Model $1^{\text {a }}$ : genotype $P$ value \\
& AA $(n=95)$ & AT $(n=236)$ & TT $(n=197)$ & $0.03^{*}$ \\
Cognitive restraint score & $13(4)$ & $12(4)$ & $11(4)$ & 0.38 \\
Disinhibition score & $6(3)$ & $7(4)$ & $6(4)$ & 0.92 \\
Hunger score & $5(3)$ & $5(4)$ & $5(4)$ & . \\
\hline
\end{tabular}

Values are mean (SD) for $n=528$. Comparisons were made using linear mixed models adjusted for age and sex. ES, effect size. ${ }^{a}$ Model 1: additive genotype model (AA versus AT versus TT). ${ }^{*}$ Significant difference between AA and TT FTO rs9939609 genotype (linear mixed model $P<0.05$ after Holm-Bonferroni correction).

3.2. FTO rs9939609 Genotype and Obesity-Related Parameters. Obesity-related parameters in men and women carrying different risk variants of FTO rs9939609 SNP are displayed in Figure 1 and Table 2. Linear mixed models for the additive genotype model revealed no significant differences in body mass, BMI, waist circumference, or waist-to-height ratio across FTO rs9939609 genotype groups (all $P \geq 0.66$; Table 2). The minor allele frequency is analogous with that expected $(0.4, \mathrm{~A})$, suggesting this is not due to an unrepresentative population. Exploratory analysis revealed no significant differences in obesity-related parameters when analysed in dominant (all $\mathrm{ES} \leq 0.13, P \geq 0.37$ ) or recessive (all ES $\leq 0.08, P \geq 0.68$ ) allele models (Supplementary Table 1).

3.3. FTO rs9939609 Genotype and Physical Activity Levels. Complete physical activity data were available for 408 participants (AA $n=74$, AT $n=177$, TT $n=157$; female: $n=83$ and male: $n=325$ ). The physical activity levels in men and women carrying different risk variants of FTO rs9939609 SNP are displayed in Table 3. Linear mixed models for the additive genotype model revealed no significant differences in physical activity levels across FTO rs 9939609 genotype groups (all $P \geq 0.10$ ). In the exploratory analysis, the dominant allele model revealed total MET minutes per week (ES $=0.22, P=0.03$, Supplementary Table 2) and a tendency for vigorous MET minutes per week (ES $=0.19, P=0.08)$ to be higher in A allele carriers (AA/AT) than nonrisk allele carriers (TT). No significant differences in moderate or walking MET minutes per week were identified in the dominant allele model (AA/AT versus TT) (all ES $\leq 0.13, P \geq 0.17$; Supplementary Table 2). The recessive allele model (AT/TT versus AA) revealed no significant differences in physical activity levels between FTO genotype groups (all ES $\leq 0.11, P \geq 0.28$; Supplementary Table 2).

3.4. FTO rs9939609 Genotype and Eating Behaviours. Eating behaviours in men and women carrying different risk variants of FTO rs9939609 SNP are displayed in Table 4. Linear 
mixed models for the additive genotype model identified a significant main effect for cognitive restraint score across FTO genotype groups $(P=0.03)$. Post hoc analysis of between-group differences revealed that the cognitive restraint score was higher in homozygous A allele carriers than AT $(\mathrm{ES}=0.25, P=0.07)$ and TT $(\mathrm{ES}=0.33, P=0.03)$ genotypes; AT and TT genotypes were similar $(\mathrm{ES}=0.06$, $P=0.49)$. No differences in disinhibition or hunger scores were seen across FTO genotype groups (both $P \geq 0.38$ ).

In the exploratory analysis, the dominant allele model (AA/AT versus TT) revealed no significant differences in cognitive restraint, disinhibition, or hunger scores between FTO genotype groups (all $\mathrm{ES} \leq 0.14, P \geq 0.12$ ). The recessive allele model was consistent with that seen in the additive genotype model, in that the cognitive restraint score was higher in homozygous $\mathrm{A}$ allele carriers (AA) than $\mathrm{T}$ allele carriers (AT/TT) $(\mathrm{ES}=0.28, P=0.01$; Supplementary Table 3). No significant differences in disinhibition or hunger scores were identified in the recessive allele model (all ES $\leq 0.08, P \geq 0.60$; Supplementary Table 3).

\section{Discussion}

The primary finding from the present study was that obesityrelated parameters were not different in physically active individuals carrying different risk variants of FTO rs9939609 SNP. Furthermore, FTO rs9939609 homozygous A allele carriers exhibited higher cognitive restraint than nonrisk allele carriers, and exploratory analysis also showed higher levels of physical activity in A allele carriers compared with nonrisk allele carriers.

The consensus of evidence suggests that FTO risk alleles are associated with elevated body weight across different ages and populations, with each minor risk allele increasing BMI and obesity risk by $0.25-0.39 \mathrm{~kg} \cdot \mathrm{m}^{-2}$ and $1.18-1.27$ fold, respectively [9]. However, the most extensively studied rs9939609 SNP had no influence on obesity-related parameters in the current study. The participants in our cohort were physically active, and the average levels of reported total and moderate- to vigorous-intensity physical activity were considerably higher in comparison with both the general population [30] and individuals in previous FTO studies [19-23]. Several studies have demonstrated, through self-reported questionnaires [31,32] and objective physical activity devices $[19,33]$, that obesity-related traits associated with FTO risk alleles are attenuated in individuals with higher physical activity levels. Therefore, it is possible that the high physical activity levels within our cohort may have diminished any differences in obesity-related parameters between FTO rs9939609 genotypes. Intriguingly, despite being a highly active group when taken as a whole, the exploratory analysis using the dominant model demonstrated that individuals with the A allele reported higher total and vigorous physical activity levels compared with TT individuals. It is possible, therefore, that this difference in physical activity patterns between FTO genotypes may further offset the adiposity-increasing effect of FTO within this cohort, but additional work is required to examine this systematically. Nonetheless, this elevation in activity was small, and these differences in physical activity levels between FTO genotypes conflict with previous studies suggesting that FTO genotype does not impact on physical activity levels [7, 31]. Contradictions between the current study and other evidence may be due to the high levels of intensive physical activity of individuals within the current study. Although Berentzen et al. [16] reported no effect of the FTO variant on physical activity in individuals classified by the authors as highly active (more than $4 \mathrm{~h}$ moderate physical activity a week), there is a lack of studies that have examined the effect of FTO genotype on activity levels in cohorts with even greater levels of physical activity. Consequently, further studies, including longitudinal investigations, are required to examine FTO-mediated differences in physical activity among individuals with varying activity status. In addition, given that much of the data on FTO-mediated differences in physical activity levels are reliant on self-reported questionnaire data, additional scientific enquiry using objective physical activity measures, such as accelerometers, is required.

It has been postulated that FTO-mediated predisposition to weight gain and obesity may be modified by dietary behaviours. Research suggests that FTO may play a significant role in the regulation of satiety and food intake [10, 11]. Furthermore, it has been reported in previous observational studies that the risk variant of FTO is associated with lower cognitive restraint and higher disinhibition and hunger, which may be indicative of poorer eating behaviours $[34,35]$. The present study extends these findings by demonstrating that physically active individuals homozygous for FTO rs9939609 risk allele display higher cognitive restraint but similar disinhibition and hunger scores compared with nonrisk allele carriers. The potential benefit of cognitive restraint on the propensity to body weight changes is highlighted by studies demonstrating a negative association between cognitive restraint and markers of adiposity (e.g., body mass and BMI) [36, 37]. However, such associations are not reported universally [38], and cognitive restraint tends to be higher in overweight than in healthy weight individuals [39]. Additionally, disinhibition has been proposed as a stronger predictor of BMI [40]. Nevertheless, increased dietary restraint during weight loss has been identified as a significant predictor of successful weight loss maintenance [41]. Therefore, it is possible that the higher cognitive restraint observed in FTO risk allele carriers in the present study may offset FTO-mediated obesity risk in physically active individuals; but further work is required to confirm this chronically.

The current study is not without limitations. The observations in the present study are limited by the crosssectional design, which precludes the ability to establish causality. Future longitudinal studies are required to establish systematically whether higher physical activity levels and cognitive restraint may diminish FTO-mediated predisposition to weight gain. In addition, the anthropometric, physical activity, and eating behaviour data were selfmeasured and self-reported using an online survey, which may have led to problems with participant measurement error, recall, and bias. Additional work is therefore required 
using more direct and objective measures. Furthermore, one of the primary measures of body fatness in the present study is BMI. Despite its frequent use in large-scale population studies, BMI does not take into account differences between fat and fat-free mass such as muscle and bone [42]. Given the current cohort reported high levels of total activity, with the majority indicating that their primary fitness goal was muscle building (52\%), it is possible that variations in body mass (and hence BMI) could be largely due to higher muscle mass. This may explain why the average BMI among individuals in the current data set falls within the overweight category. Consequently, future studies using more direct measures of body adiposity are required to replicate the current study's findings. Finally, statistical power was limited in the dominant and recessive models analysed in this paper. However, the results from these exploratory models allow for preliminary inferences that may guide a larger population-based study.

\section{Conclusion}

Within a physically active cohort, risk allele carriers of FTO rs9939609 exhibited greater physical activity levels and cognitive restraint than nonrisk allele carriers despite demonstrating similar adiposity-related measures. Future studies with repeated and objective measurements are required to further investigate physical activity and dietary behaviours that underscore the effects of FTO on obesity risk.

\section{Conflicts of Interest}

Nathan R. West, Nicola C. Hanson, Samantha E. Decombel, and Stuart J. Grice work for FitnessGenes Ltd.

\section{Authors' Contributions}

Nathan R. West and James Dorling contributed equally to this work.

\section{Acknowledgments}

The authors thank the volunteers for their participation in this study. This study was funded by FitnessGenes Ltd., Bicester OX26 4LD, UK.

\section{Supplementary Materials}

Supplementary Methods: ethnicity information collection. Supplementary Table 1: obesity-related parameters in men and women carrying different risk variants of the FTO rs9939609 single nucleotide polymorphism. Supplementary Table 2: physical activity levels in men and women carrying different risk variants of the FTO rs9939609 single nucleotide polymorphism. Supplementary Table 3: eating behaviour in men and women carrying different risk variants of the FTO rs9939609 single nucleotide polymorphism. (Supplementary Materials)

\section{References}

[1] M. Ng, T. Fleming, M. Robinson et al., "Global, regional, and national prevalence of overweight and obesity in children and adults during 1980-2013: a systematic analysis for the Global Burden of Disease Study 2013," The Lancet, vol. 384, no. 9945, pp. 766-781, 2014.

[2] A. A. van der Klaauw and I. S. Farooqi, "The hunger genes: pathways to obesity," Cell, vol. 161, no. 1, pp. 119-132, 2015.

[3] T. M. Frayling, N. J. Timpson, M. N. Weedon et al., "A common variant in the FTO gene is associated with body mass index and predisposes to childhood and adult obesity," Science, vol. 316, no. 5826, pp. 889-894, 2007.

[4] J. K. Hertel, S. Johansson, H. Raeder et al., "Genetic analysis of recently identified type 2 diabetes loci in 1,638 unselected patients with type 2 diabetes and 1,858 control participants from a Norwegian population-based cohort (the HUNT study)," Diabetologia, vol. 51, no. 6, pp. 971-977, 2008.

[5] K. Hotta, Y. Nakata, T. Matsuo et al., "Variations in the FTO gene are associated with severe obesity in the Japanese," Journal of Human Genetics, vol. 53, no. 6, pp. 546-553, 2008.

[6] M. Villalobos-Comparán, M. Teresa Flores-Dorantes, M. Teresa Villarreal-Molina et al., "The FTO gene is associated with adulthood obesity in the Mexican population," Obesity, vol. 16, no. 10, pp. 2296-2301, 2008.

[7] G. Liu, H. Zhu, V. Lagou et al., "FTO variant rs9939609 is associated with body mass index and waist circumference, but not with energy intake or physical activity in European- and African-American youth," BMC Medical Genetics, vol. 11, p. 57, 2010.

[8] Y. Sun, J. Sun, X. Wang, W. You, and M. Yang, "Variants in the fat mass and obesity associated (FTO) gene are associated with obesity and C-reactive protein levels in Chinese Han populations," Clinical and Investigative Medicine, vol. 33, no. 6, pp. E405-E412, 2010.

[9] R. J. F. Loos and G. S. H. Yeo, "The bigger picture of FTO: the first GWAS-identified obesity gene," Nature Reviews Endocrinology, vol. 10, no. 1, pp. 51-61, 2014.

[10] J. M. McCaffery, G. D. Papandonatos, I. Peter et al., "Obesity susceptibility loci and dietary intake in the Look AHEAD Trial," American Journal of Clinical Nutrition, vol. 95, no. 6, pp. 1477-1486, 2012.

[11] Q. Qi, T. O. Kilpeläinen, M. K. Downer et al., "FTO genetic variants, dietary intake and body mass index: insights from 177,330 individuals," Human Molecular Genetics, vol. 23, no. 25, pp. 6961-6972, 2014.

[12] J. Wardle, S. Carnell, C. M. Haworth, I. S. Farooqi, S. O'Rahilly, and R. Plomin, "Obesity associated genetic variation in FTO is associated with diminished satiety," Journal of Clinical Endocrinology and Metabolism, vol. 93, no. 9, pp. 3640-3643, 2008.

[13] M. Tanofsky-Kraff, J. C. Han, K. Anandalingam et al., "The FTO gene rs9939609 obesity-risk allele and loss of control over eating," American Journal of Clinical Nutrition, vol. 90, no. 6, pp. 1483-1488, 2009.

[14] E. Karra, O. G. O’Daly, A. I. Choudhury et al., "A link between FTO, ghrelin, and impaired brain food-cue responsivity," Journal of Clinical Investigation, vol. 123, no. 8, pp. 3539-3551, 2013.

[15] J. R. Speakman, K. A. Rance, and A. M. Johnstone, "Polymorphisms of the FTO gene are associated with variation in energy intake, but not energy expenditure," Obesity, vol. 16, no. 8, pp. 1961-1965, 2008.

[16] T. Berentzen, S. I. Kring, C. Holst et al., "Lack of association of fatness-related FTO gene variants with energy expenditure or 
physical activity," Journal of Clinical Endocrinology and Metabolism, vol. 93, no. 7, pp. 2904-2908, 2008.

[17] E. Corpeleijn, L. Petersen, C. Holst et al., "Obesity-related polymorphisms and their associations with the ability to regulate fat oxidation in obese Europeans: the NUGENOB study," Obesity, vol. 18, no. 7, pp. 1369-1377, 2010.

[18] C. H. Andreasen, K. L. Stender-Petersen, M. S. Mogensen et al., "Low physical activity accentuates the effect of the FTO rs9939609 polymorphism on body fat accumulation," $D i$ abetes, vol. 57, no. 1, pp. 95-101, 2008.

[19] C. Celis-Morales, C. F. Marsaux, K. M. Livingstone et al., "Physical activity attenuates the effect of the FTO genotype on obesity traits in European adults: the Food4Me study," Obesity, vol. 24, no. 4, pp. 962-969, 2016.

[20] J. Y. Kim, J. T. DeMenna, S. Puppala et al., "Physical activity and FTO genotype by physical activity interactive influences on obesity," BMC Genetics, vol. 17, no. 1, p. 47, 2016.

[21] T. O. Kilpeläinen, L. Qi, S. Brage et al., "Physical activity attenuates the influence of FTO variants on obesity risk: a meta-analysis of 218,166 adults and 19,268 children," PLoS Medicine, vol. 8, no. 11, p. e1001116, 2011.

[22] J. A. Mitchell, T. S. Church, T. Rankinen, C. P. Earnest, X. Sui, and S. N. Blair, "FTO genotype and the weight loss benefits of moderate intensity exercise," Obesity, vol. 18, no. 3, pp. 641643, 2010.

[23] T. Rankinen, T. Rice, M. Teran-Garcia, D. C. Rao, and C. Bouchard, "FTO genotype is associated with exercise training-induced changes in body composition," Obesity, vol. 18, no. 2, pp. 322-326, 2010.

[24] National Heart, Lung, and Blood Institute and North American Association for the Study of Obesity, Practical Guide on the Identification, Evaluation, and Treatment of Overweight and Obesity in Adults, Bethesda, MD,: National Institutes of Health. NIH publication No. 00-4084; Bethesda, MD, USA, October, 2000.

[25] C. L. Craig, A. L. Marshall, M. Sjöström et al., "International physical activity questionnaire: 12-country reliability and validity," Medicine and Science in Sports and Exercise, vol. 35, no. 8, pp. 1381-1395, 2003.

[26] A. J. Stunkard and S. Messick, "The three-factor eating questionnaire to measure dietary restraint, disinhibition and hunger," Journal of Psychosomatic Research, vol. 29, no. 1, pp. 71-83, 1985.

[27] A. D. Skol, L. J. Scott, G. R. Abecasis, and M. Boehnke, “Joint analysis is more efficient than replication-based analysis for two-stage genome-wide association studies," Nature Genetics, vol. 38, no. 2, pp. 209-213, 2006.

[28] R. A. Armstrong, "When to use the Bonferroni correction," Ophthalmic and Physiological Optics, vol. 34, no. 5, pp. 502508, 2014.

[29] J. Cohen, Statistical Power Analysis for the Behavioural Sciences, pp. 22-25, Lawrence Erlbaum Associates, Hillsdale, NJ, USA, 1988.

[30] A. Loyen, L. Van Hecke, M. Verloigne et al., "Variation in population levels of physical activity in European adults according to cross-European studies: a systematic literature review within DEDIPAC," International Journal of Behavioral Nutrition and Physical Activity, vol. 13, no. 1, p. 72, 2016.

[31] M. Hakanen, O. T. Raitakari, T. Lehtimäki et al., "FTO genotype is associated with body mass index after the age of seven years but not with energy intake or leisure-time physical activity," Journal of Clinical Endocrinology and Metabolism, vol. 94, no. 4, pp. 1281-1287, 2009.
[32] E. Sonestedt, C. Roos, B. Gullberg, U. Ericson, E. Wirfält, and M. Orho-Melander, "Fat and carbohydrate intake modify the association between genetic variation in the FTO genotype and obesity," American Journal of Clinical Nutrition, vol. 90, no. 5, pp. 1418-1425, 2009.

[33] J. R. Ruiz, I. Labayen, F. B. Ortega et al., "Attenuation of the effect of the FTO rs9939609 polymorphism on total and central body fat by physical activity in adolescents: the HELENA study," Archives of Pediatrics and Adolescent Medicine, vol. 164, no. 4, pp. 328-333, 2010.

[34] M. C. Cornelis, E. B. Rimm, G. C. Curhan et al., "Obesity susceptibility loci and uncontrolled eating, emotional eating and cognitive restraint behaviors in men and women," Obesity, vol. 22, no. 5, pp. E135-E141, 2014.

[35] J. Harbron, L. van der Merwe, M. G. Zaahl, M. J. Kotze, and M. Senekal, "Fat mass and obesity-associated (FTO) gene polymorphisms are associated with physical activity, food intake, eating behaviours, psychological health, and modeled change in body mass index in overweight/obese Caucasian adults," Nutrients, vol. 6, no. 8, pp. 3130-3152, 2014.

[36] G. D. Foster, T. A. Wadden, R. M. Swain, A. J. Stunkard, P. Platte, and R. A. Vogt, "The Eating Inventory in obese women: clinical correlates and relationship to weight loss," International Journal of Obesity and Related Metabolic Disorders, vol. 22, no. 8, pp. 778-785, 1998.

[37] V. Hainer, M. Kunesova, F. Bellisle et al., "The Eating Inventory, body adiposity and prevalence of diseases in a quota sample of Czech adults," International Journal of Obesity, vol. 30, no. 5, pp. 830-836, 2006.

[38] V. Provencher, V. Drapeau, A. Tremblay, J. P. Després, and S. Lemieux, "Eating behaviours and indexes of body composition in men and women from the Québec family study," Obesity Research, vol. 11, no. 6, pp. 783-792, 2003.

[39] J. C. Cappelleri, A. G. Bushmakin, R. A. Gerber et al., "Psychometric analysis of the Three-Factor Eating Questionnaire-R21: results from a large diverse sample of obese and non-obese participants," International Journal of Obesity, vol. 33, no. 6, pp. 611-620, 2009.

[40] A. Lesdéma, G. Fromentin, J. J. Daudin et al., "Characterization of the Three-Factor Eating Questionnaire scores of a young French cohort," Appetite, vol. 59, no. 2, pp. 385-390, 2012.

[41] N. Vogels, K. Diepvens, and M. S. Westerterp-Plantenga, "Predictors of long-term weight maintenance," Obesity Research, vol. 13, no. 12, pp. 2162-2168, 2005.

[42] K. J. Rothman, "BMI-related errors in the measurement of obesity," International Journal of Obesity, vol. 32, pp. S56-S59, 2008. 


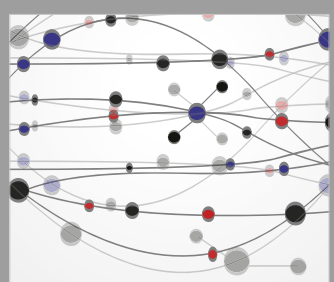

The Scientific World Journal
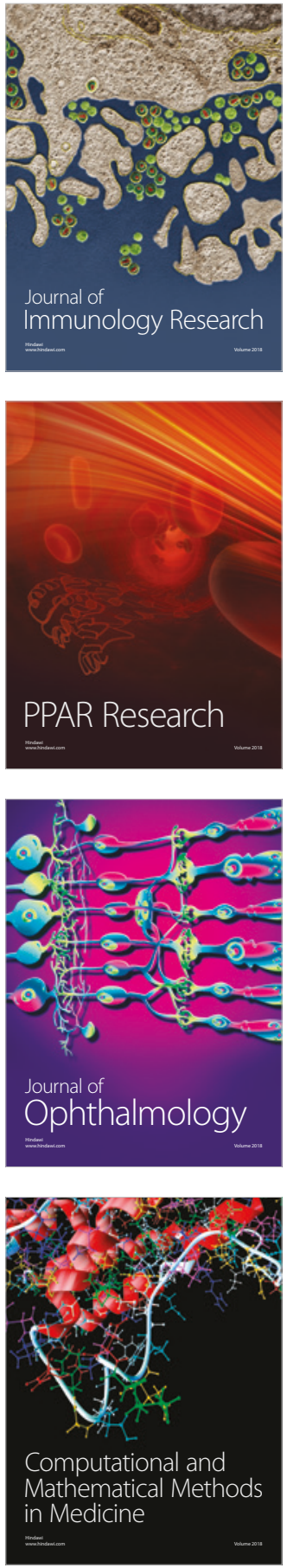

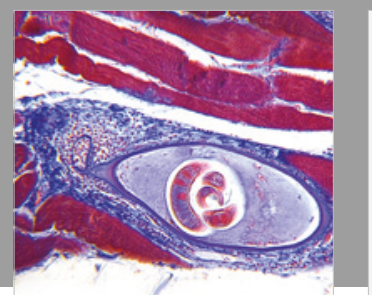

Gastroenterology Research and Practice

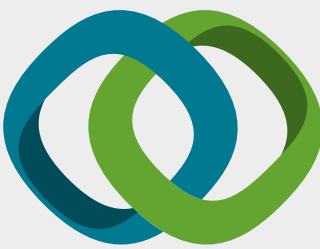

\section{Hindawi}

Submit your manuscripts at

www.hindawi.com
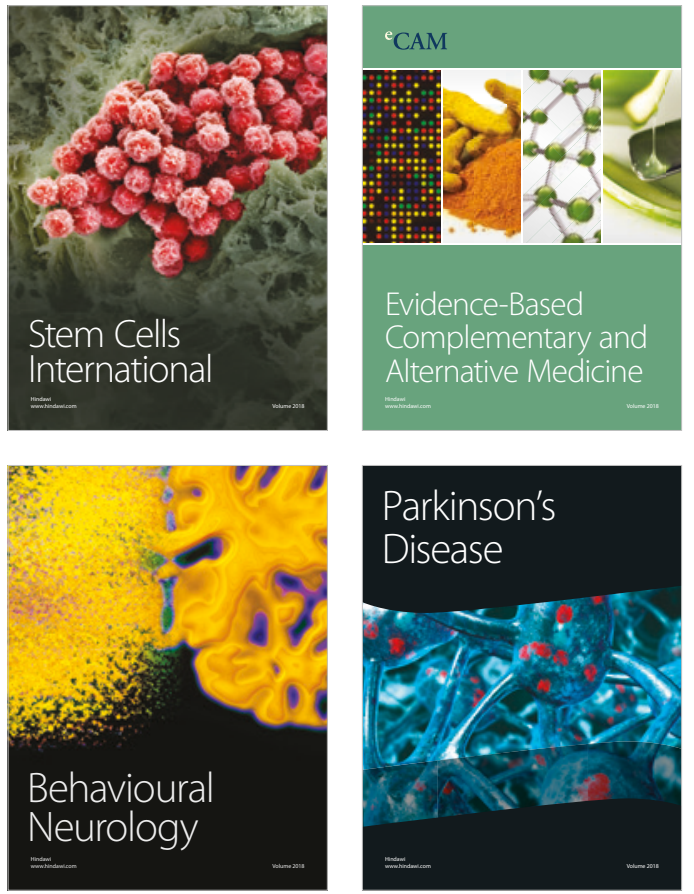

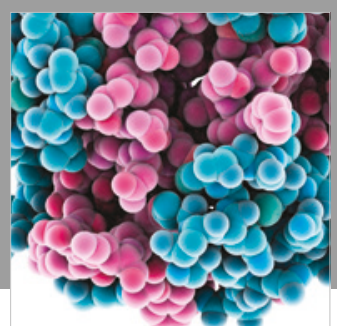

ournal of

Diabetes Research

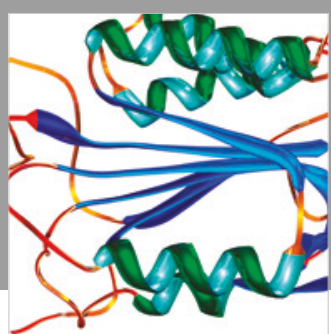

Disease Markers
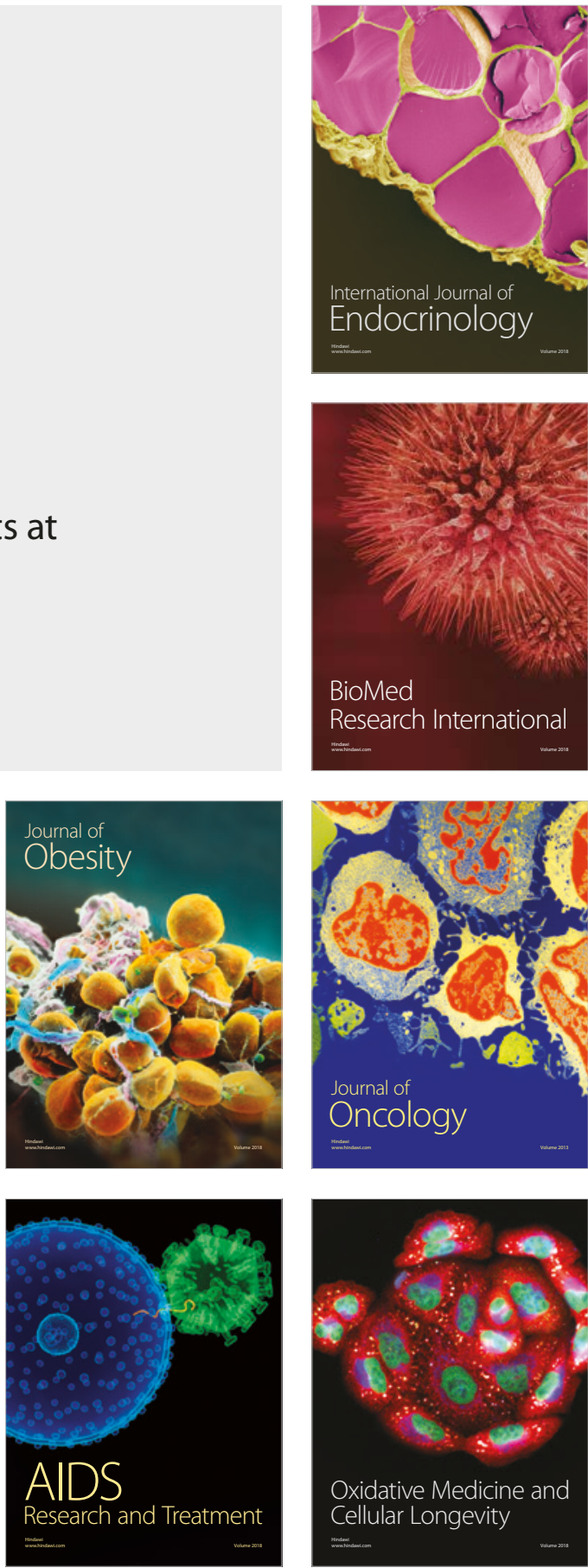